\title{
The ASA score predicts infections, cardiovascular complications, and hospital readmissions after hip fracture - A nationwide cohort study
}

\author{
A. C. Meyer ${ }^{1}$ (D) H. Eklund ${ }^{1} \cdot$ M. Hedström ${ }^{2,3} \cdot$ K. Modig $^{1}$
}

Received: 7 January 2021 / Accepted: 7 April 2021 / Published online: 19 May 2021

(C) The Author(s) 2021

\begin{abstract}
Summary This study examines the association between the ASA physical status classification score at hip fracture surgery and severe postoperative complications in patients aged 60 and older. Among both men and women, ASA scores consistently predict a wide range of complications including infections, cardiovascular complications, hospital readmissions, and death.

Introduction Hip fractures are common in aging populations and associated with poor prognosis. This study examines how the American Society of Anaesthesiologists (ASA) physical status classification is related to severe complications among hip fracture patients including infections, cardiovascular diseases, hospital readmissions, and death.

Methods Based on a linkage of the Swedish National Inpatient Register with the Swedish National Registry for Hip Fractures (RIKSHÖFT), this study includes patients aged 60+ with first hip fracture between 1998 and 2017. We estimated associations between ASA score and complications during the hospital stay and during 1 year after hip fracture using multivariable-adjusted logistic regression and Cox proportional hazard regression.

Results The study population included 170,193 hip fracture patients of which $24 \%$ died and $39 \%$ were readmitted to hospital within 1 year. The most common complications were urinary tract infections, pneumonia, second hip fractures, and heart failure. Among both men and women, higher ASA scores were consistently associated with higher risks for all complications included in this study. The strongest associations were observed for heart failure, myocardial infarction, pneumonia, and death.

Conclusion ASA scores are routinely assessed in clinical practice and predict a wide range of postoperative complications among hip fracture patients. Since many complications may be preventable through adequate drug treatment, rehabilitation, and risk awareness, future studies should examine the mechanisms linking ASA scores to complication risk in order to improve preventive strategies. Particularly, the high risk of cardiovascular complications among patients with high ASA scores deserves clinical and scientific attention.
\end{abstract}

Keywords ASA score $\cdot$ Comorbidity $\cdot$ Complications $\cdot$ Hip fracture $\cdot$ Prognosis $\cdot$ Readmission

A. C. Meyer

anna.meyer@ki.se

1 Unit of Epidemiology, Institute of Environmental Medicine, Karolinska Institutet, PO Box 210, SE-17177 Stockholm, Sweden

2 Department of Clinical Science, Intervention and Technology (CLINTEC) Karolinska Institutet, SE-17177 Stockholm, Sweden

3 Department of Orthopaedics, Karolinska University Hospital, Stockholm, Sweden

\section{Introduction}

In Sweden, the lifetime risk of sustaining a hip fracture is approximately $20 \%$ among women and $10 \%$ among men [1]. Hip fractures often affect frail older patients for which complications, such as infections, can be life-threatening. As a result, one in four patients dies within 1 year [2]. Identifying individuals at risk of serious complications is thus of great interest for patients and clinicians.

In clinical practice, patients' general health and comorbidity level at the time of surgery are routinely assessed through the American Society for of Anaesthesiologists (ASA) physical status classification [3]. Although ASA scores reflect a subjective assessment of patients' comorbidity level, they 
have been shown to predict both mortality [4] and complications among hip fracture patients, such as urinary tract infections (UTI) and postoperative pneumonia [5-7]. A systematic review concluded that ASA scores were more robust predictors of hospital readmissions after hip fracture than other measures of comorbidity, for instance the Charlson comorbidity index (CCI) or a set of individual comorbidities [8]. Even though several studies investigated the association between ASA scores and hospital readmissions among hip fracture patients [8], none have, to our knowledge, examined the risk of cause-specific readmissions due to infections or cardiovascular complications.

Hip fracture patients are at higher risk of serious cardiovascular events such as myocardial infarction and stroke than the general population [9-11]. Studies have also shown that higher comorbidity levels, albeit not measured through the ASA score, further increase the risk of these events among hip fracture patients $[10,11]$. In this nationwide cohort study, we aimed to explore how ASA scores are related to postoperative complications among hip fracture patients in Sweden. We examine the occurrence of common complications with a large clinical impact including infections, cardiovascular diseases, and hospital readmissions.

\section{Materials and methods}

\section{Data sources and study population}

This study was based on a linkage of the Swedish National Inpatient Register (NPR), Cause of Death Register, and the Swedish National Registry for Hip Fractures RIKSHÖFT (SRH) using the unique personal identification number assigned to all Swedish residents. The NPR covers all hospital admissions within Sweden since 1987 including primary and contributing causes for hospitalization assigned by physicians [12]. The SRH is a clinical register containing medical information on approximately $80 \%$ of hip fractures in Sweden [13].

We included all patients aged 60 or older with first hip fracture between 1998 and 2017 registered in the SRH. Patients with hip fracture diagnoses in the NPR within 10 years prior to their index fracture were excluded (International Classification of Diseases (ICD)-9: 820 or ICD-10: S72.0-72.2 as primary diagnosis for hospital admission). We further excluded patients with pathological fractures (1.7\%), missing information on ASA score $(8.0 \%)$, other covariates $(0.1 \%)$, or ASA score 5 , i.e., moribund patients $(0.1 \%$, Fig. S1). Analyses of complications during the hospital stay were restricted to a subset of patients, since the documentation of postoperative infections in the SRH was not conducted throughout the entire study period and in all hospitals. We restricted these analyses to patients treated after 2010 in hospitals which documented the presence or absence of infections in at least $95 \%$ of their patients' files. Since postoperative complications such as UTI and pneumonia were recorded in a separate form, the study population for these outcomes was further restricted to hospitals that used this form for at least $95 \%$ of patients. In total, information on complications during index hospitalization was available for $30.1 \%$ (superficial wound infections) and $8.5 \%$ (UTI and pneumonia) of patients.

\section{Variables and definitions}

The ASA scores, assigned by anesthetists at the time of surgery, were retrieved from the SRH and ranged from 1 characterizing a healthy patient to 4 characterizing a patient with severe comorbidity [3]. A description of each ASA score is provided in Table S1. Outcomes were measured at two occasions, during the hospital stay and within 1 year of the fracture. Complications during the hospital stay were reported by clinicians to the SRH through questionnaires which included superficial wound infections, pneumonia and UTI.

Complications within 1 year after hip fracture were identified in the NPR and included pneumonia, pulmonary embolism, myocardial infarction, stroke, heart failure, second hip fractures, hospital readmissions for any reason and death. We defined these complications through the following ICD-10 codes as primary causes for hospitalization: I11.0 and I50 for heart failure, I21-I22 for myocardial infarction, I26 for pulmonary embolism, I61-I64 for stroke, and S72.0-72.2 for hip fracture. Pneumonia was identified through the ICD-10 codes $\mathrm{J} 12-15$, J18, and $\mathrm{J} 69$ occurring as primary or secondary diagnosis in the NPR. In order to distinguish second hip fractures from readmissions for the index fracture, we applied a minimum period of 14 days between the first and second fracture. This strategy is in accordance with a previous study on identifying hip fractures in the Swedish NPR [13]. For all other outcomes except for death, at least one night between a patient's discharge and readmission was required in order to distinguish readmissions from transfers between clinics. Dates of death were extracted from the Cause of Death Register and patients were at risk for this outcome from the time of their hip fracture.

Covariates included age at hip fracture, fracture year, type of fracture, and surgical method. To account for potentially non-linear associations, we included age and fracture year as categorical variables divided into 5-year intervals with patients older than 95 years categorized into one age group. We distinguished six fracture types: non-displaced intracapsular fractures, displaced intracapsular fractures, basicervical fractures, pertrochanteric fractures with two fragments, pertrochanteric fractures with more than two fragments, and subtrochanteric fractures. Surgical methods were divided into nail or screw only, nail or screw with side plate, intramedullary nail, partial or total hip replacement, and other treatment. 


\section{Statistics}

Bivariate associations between ASA scores and all outcomes were presented in tables. To examine the association between ASA score and complications during the hospital stay, we estimated odds ratios (OR) with $95 \%$ confidence intervals (CI) using logistic regression. For the complications during the first year after the fracture, we employed Cox proportional hazard regression with age as the underlying time scale to estimate hazard ratios (HR) with $95 \%$ CIs censoring individuals at the time of their death or the end of follow-up on December, 31st 2017. ASA score 2 was chosen as the reference category and all regression models were adjusted for age and fracture year. In addition, we estimated models adjusted for fracture type and surgical method. However, since neither fracture type nor surgical method affected the estimates notably, these variables were not included in the final models but are instead presented in supplementary tables.

The results of this study indicated a strong relationship between ASA score and cardiovascular complications. In order to determine to what extent the increased risk of complications among patients with high ASA scores is driven by preexisting cardiovascular comorbidities, we conducted sensitivity analyses adjusting for patients' history of cardiovascular disease including myocardial infarction, stroke, and heart failure during 10 years before the index fracture. Furthermore, we conducted sensitivity analyses reducing the follow-up time from 1 year to 30 days after hip fracture in order to examine whether ASA scores are associated with short-term complications.

The proportionality of hazards ( $\mathrm{PH}$ ) assumption was tested using Schoenfeld residuals and log-log plots. All analyses were conducted for men and women separately and for both sexes together using Stata version 16.

\section{Results}

The study population included 170,193 patients with first hip fracture, $68.8 \%$ of which were women. Characteristics of the study population are shown in Table 1. Advanced age and male sex were associated with higher ASA scores. The risk of death was strongly correlated with ASA score. Among patients with ASA score 1, 7.0\% died within 1 year of their fracture while almost half of patients (48\%) with ASA score 4 died. Altogether, $39.0 \%$ of the study population was readmitted to hospital for any reason within 1 year of the fracture, 5.3\% hospitalized with pneumonia, $4.9 \%$ with a second hip fracture, and $2.6 \%$ with heart failure. Stroke, myocardial infarction, and pulmonary embolism each affected less than $2 \%$ of patients. Among

Table 1 Study population stratified by ASA score $(N=170,193)$

\begin{tabular}{|c|c|c|c|c|}
\hline & ASA $1 n=11,500$ & ASA $2 n=66,095$ & ASA $3 n=78,396$ & ASA $4 n=14,202$ \\
\hline \multicolumn{5}{|l|}{ Age, $n(\%)$} \\
\hline $60-69$ years & $2783(24.2)$ & $6702(10.1)$ & $4843(6.2)$ & $963(6.8)$ \\
\hline 70-79 years & $3698(32.2)$ & $16,866(25.5)$ & $16,509(21.1)$ & $3155(22.2)$ \\
\hline $80-89$ years & $3844(33.4)$ & $30,933(46.8)$ & $39,328(50.2)$ & $6920(48.7)$ \\
\hline 90 years and older & $1175(10.2)$ & $11,594(17.5)$ & $17,716(22.6)$ & $3164(22.3)$ \\
\hline Male $(\%)$ & $3220(28.0)$ & $17,349(26.3)$ & $26,708(34.1)$ & $5855(41.2)$ \\
\hline \multicolumn{5}{|c|}{ Complications during index hospitalization ${ }^{\mathrm{a}}$} \\
\hline Superficial wound infections (\%) & $15(0.6)$ & $193(1.0)$ & $315(1.2)$ & $44(1.1)$ \\
\hline UTI $(\%)$ & $55(6.3)$ & $468(7.9)$ & $690(10.1)$ & $80(9.6)$ \\
\hline Pneumonia (\%) & $10(1.1)$ & $197(3.3)$ & $368(5.4)$ & $79(9.5)$ \\
\hline \multicolumn{5}{|c|}{ Complications during the first year after fracture } \\
\hline Readmission (any reason) $(\%)$ & $2993(26.0)$ & $23,282(35.2)$ & $33,867(43.2)$ & $6191(43.6)$ \\
\hline Pneumonia $(\%)$ & $270(2.4)$ & $2514(3.8)$ & $5152(56.6)$ & $1113(7.8)$ \\
\hline Pulmonary embolism (\%) & $72(0.6)$ & $520(0.8)$ & $697(0.9)$ & $126(0.9)$ \\
\hline Myocardial infarction (\%) & $77(0.7)$ & $616(0.9)$ & $1075(1.4)$ & $282(2.0)$ \\
\hline Stroke $(\%)$ & $125(1.1)$ & $1096(1.7)$ & $1704(2.2)$ & $227(1.6)$ \\
\hline Heart failure $(\%)$ & $81(0.7)$ & $946(1.4)$ & $2621(3.3)$ & $729(5.1)$ \\
\hline Second hip fracture $(\%)$ & $409(3.6)$ & $3123(4.7)$ & $4150(5.3)$ & $697(4.9)$ \\
\hline Death $(\%)$ & $804(7.0)$ & 9955 (15.1) & $23,229(29.6)$ & $6821(48.0)$ \\
\hline
\end{tabular}

ASA American Society for Anaesthesiologists physical status classification, UTI urinary tract infections

${ }^{a}$ Complications during index hospitalization observed among subset of patients treated in hospitals that recorded these outcomes $(N=51,212$ for superficial wound infections; $N=14,469$ for UTI and pneumonia) 
those with information about complications during index hospitalization, $8.9 \%$ experienced UTI, $4.5 \%$ pneumonia, and $1.1 \%$ superficial wound infections. Higher ASA scores were associated with higher risks for all complications both during index hospitalization and within 1 year of sustaining the hip fracture despite patients' substantially higher mortality and, as a result, shorter time at risk.

The association between ASA score and complications during index hospitalization adjusted for age and fracture year are presented in Fig. 1 and Table S2. Compared to the reference group, ASA score 3 was associated with higher odds of all complications, while an ASA score of 4 was only associated with higher odds of pneumonia. Patients categorized as ASA 1 had lower odds of superficial wound infections and pneumonia, but not UTI. The association was strongest for pneumonia; the odds to develop pneumonia were almost 3 times higher among individuals with ASA score 4 than in the reference group (OR [95\%CI]: 2.87 [2.19; 3.78]).

Figure 2 and Table S3 show the relationship between ASA score and complications during 1 year after hip fracture. We found associations between ASA score and all outcomes following consistent dose-response patterns; higher ASA scores were associated with higher hazards to develop all complications. The largest relative effect sizes were observed for mortality and heart failure. Individuals categorized as ASA 4 were at five-fold elevated risk to suffer from heart failure $(\mathrm{HR}=$ 5.00 [CI 4.54-5.51]) and four-fold elevated risk of dying (HR $=4.28$ [CI 4.15-4.41]) at any point during the first year after hip fracture compared to individuals with ASA score 2. Relative effect sizes were smallest for second hip fractures, stroke, and pulmonary embolism although HR still indicated elevated hazards for individuals with higher ASA scores. We did not detect notable sex differences and all associations remained unchanged when adjusting for fracture type and surgery method in a stepwise manner (Table S3).

Our sensitivity analyses indicated that part of the elevated hazards of cardiovascular complications as well as mortality can be attributed to a history of cardiovascular diseases among patients with higher ASA scores (Table S4). HR were particularly attenuated for heart failure, although three-fold increased hazards among patients with the highest ASA score compared to the reference remained even after adjustment ( $\mathrm{HR}=3.20$ [CI 2.90-3.54]). Adjusting for cardiovascular disease history did not affect the association between ASA score and readmissions to hospital for any reason, pneumonia, pulmonary embolism, or recurrent hip fractures. The associations between ASA scores and complications during 30 days after hip fracture were similar to our main findings with regards to both direction and magnitude (Table S5).

Statistical tests of scaled Schoenfeld residuals indicated violations of the $\mathrm{PH}$ assumption in several models but, due to the size of this study, even minor violations reach statistical significance. Visual examinations of log-log plots indicated no cross-over of hazard curves and reasonably parallel patterns. Moreover, restricting the follow-up to time periods under which the $\mathrm{PH}$ assumption was statistically met did not alter the results notably.

\section{Discussion}

In this large register-based study, we show that hip fracture patients with higher ASA scores are at elevated risk of various severe complications including infections, hospital
Fig. 1 Odds ratios and $95 \%$ confidence intervals for complications during index hospitalization. Adjusted for age and fracture year. $N=51,212$ for superficial wound infections; $N=$ 14,469 for UTI and pneumonia. ASA: American Society for Anaesthesiologists physical status classification; UTI: urinary tract infections

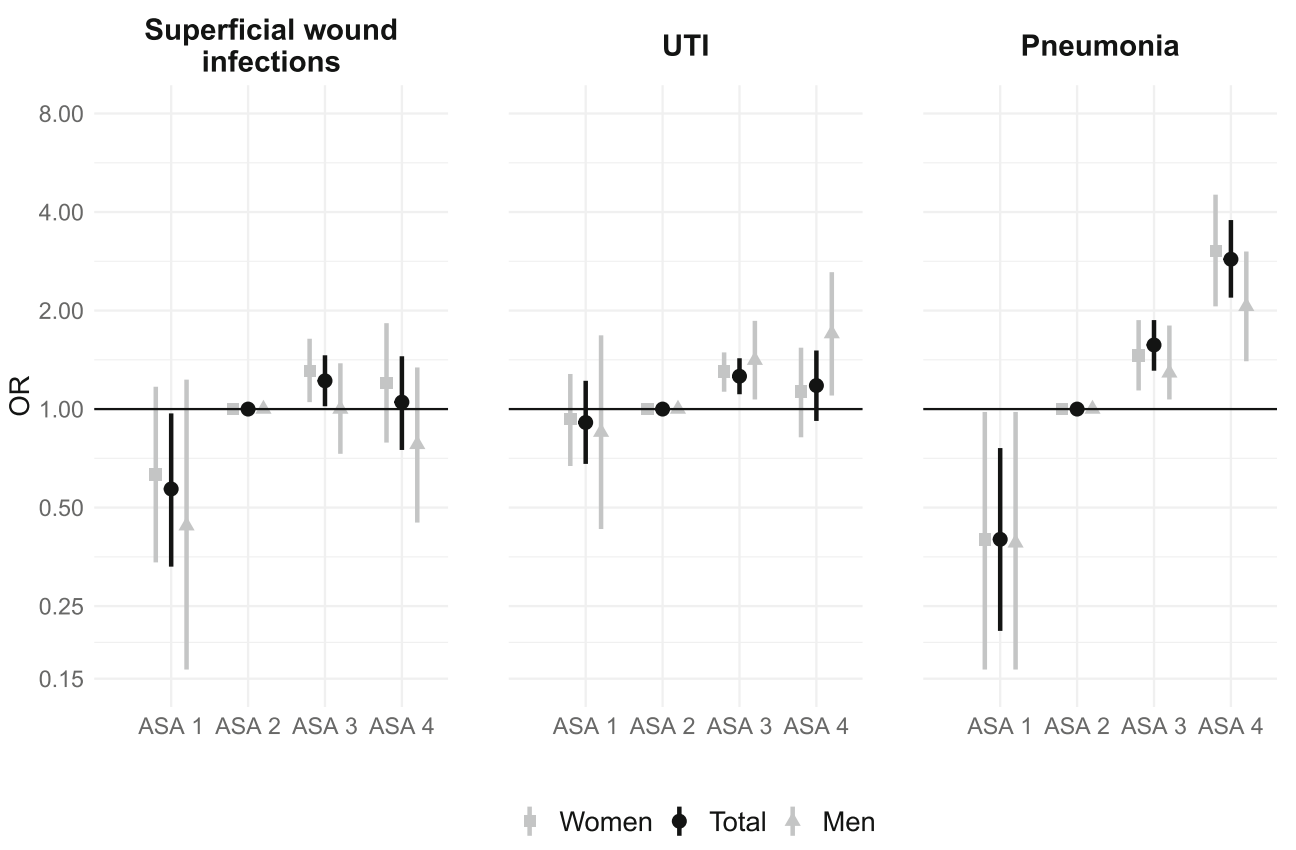




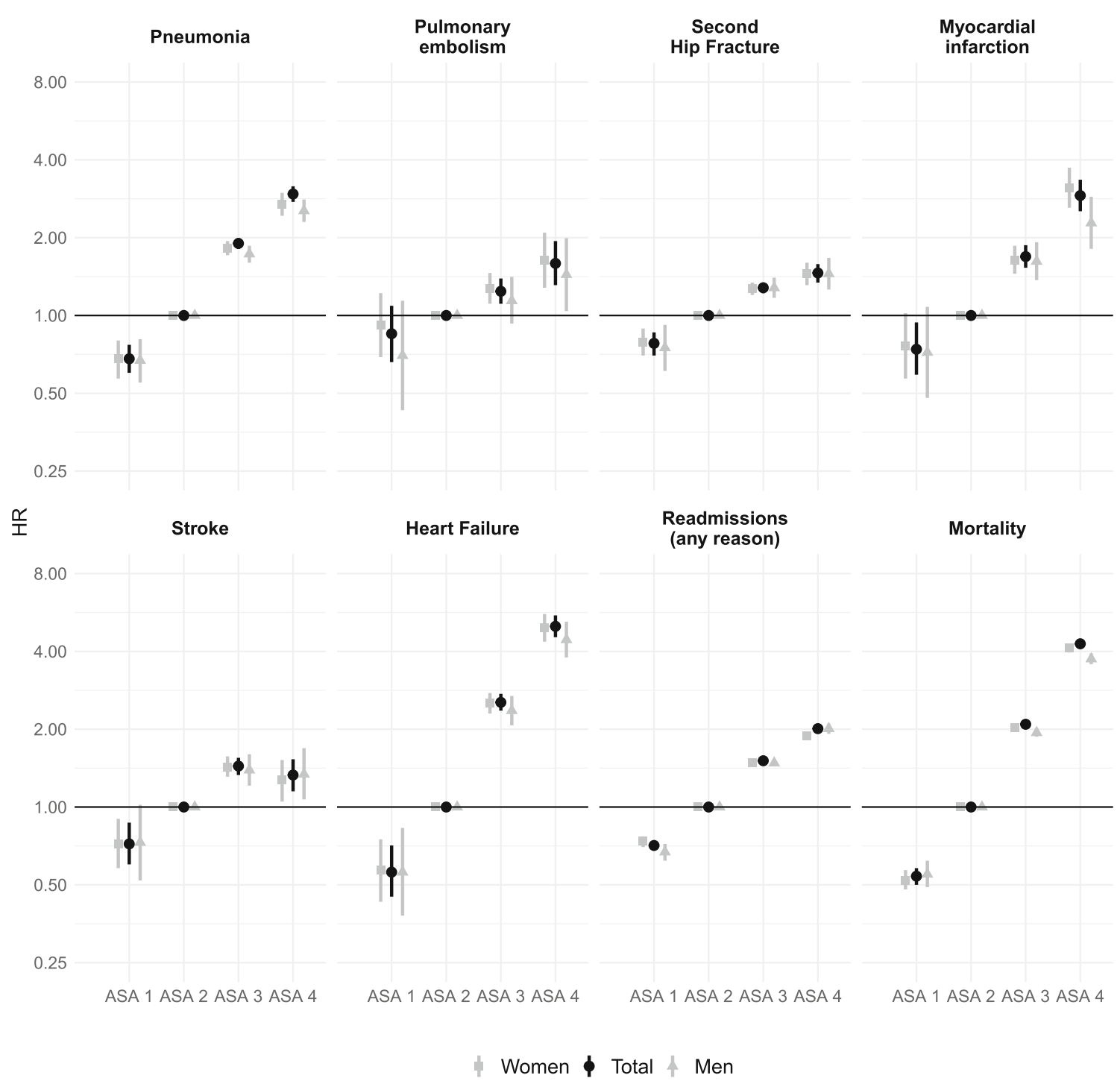

Fig. 2 Hazard ratios and 95\% confidence intervals for complications resulting in readmission to hospital during 1 year after hip fracture. Adjusted for age and fracture year. $N=170,193$. ASA: American Society for Anaesthesiologists physical status classification

readmissions, cardiovascular diseases, and death. Higher ASA scores were consistently associated with complications both during the index hospitalization and during the year after hip fracture. We found particularly strong associations between higher ASA scores and higher risks of cardiovascular complications, which could only partly be explained by patients' history of cardiovascular disease. Moreover, we found no apparent sex differences in the relationship between ASA and any of the complications included in this study. This suggests that the ASA score is equally predictive for complications among men and women although male hip fracture patients have higher comorbidity levels and higher mortality rates than women [2].

In line with our findings, previous research demonstrated that a higher comorbidity burden is associated with an increased risk of complications among hip fracture patients such as UTI [6, 14], pneumonia [7, 14], pulmonary embolism [4], hospital readmissions [8, 15-17], cardiovascular events [9-11, 14], and mortality [4, 18]. While the relationship between comorbidities and postoperative complications after hip fracture is well established, our work is to date the largest study linking ASA scores to a wide range of postoperative complications both during hip fracture hospitalizations and after discharge. In contrast to many previous studies, the size of our study allowed to distinguish between four ASA levels rather than aggregating scores into a dichotomous variable. We found that already ASA score 2 is associated with a substantially higher risk of many severe complications compared to the lowest ASA level. To our knowledge, this is also the first study comparing the risk of cardiovascular complications among patients with different ASA scores.

ASA scores are routinely assessed and readily available in clinical practice, but its limitations including subjectivity when assigning scores should be considered $[19,20]$. A 
previous study has shown a decent interrater reliability between clinicians assigning ASA scores to orthopedic trauma patients; yet, it is far from perfect [20]. Still, our findings as well as previous research suggest that the ASA classification is a surprisingly robust predictor of post-operative complications in older hip fracture patients. In a systematic review, Ali and Gibbons concluded that ASA scores were superior predictors of re-admissions among hip fracture patients than other comorbidity measures, such as the CCI [8]. Moreover, Doherty et al. showed in a recent study that ASA scores performed equally well in predicting post-operative complications as the more complex Nottingham Hip Fracture Score [14]. In contrast, Ji et al. found that other measures such as grip strength can predict in-hospital complications more reliably than ASA score [21]. However, in their study, ASA scores were dichotomized hence losing part of their discriminative abilities.

Older individuals with hip fracture are at elevated risk of cardiovascular diseases $[10,11]$ and our study indicated that patients with high ASA scores are particular risk groups. We further found that this is partly explained by pre-fracture cardiovascular disease, which likely affected the allocation of patients to higher ASA scores. On the other hand, one can speculate that the larger comorbidity burden among patients with high ASA scores together with the trauma itself followed by surgery and postoperative immobilization results in a catabolic state [22] and has a negative impact on pre-existing chronic conditions. Either way, the high risk of myocardial infarction and heart failure among hip fracture patients with higher ASA scores should be acknowledged and deserves clinical attention.

Our study has a number of strengths and limitations. It is possible that some of the complications measured during the index hospitalization were already present at the time of the hip fracture. In our data, we could not determine whether inhospital complications occurred after the hip fracture or were already present at the time of hospital admission. Pneumonia specifically may have contributed to the assignment of high ASA scores among these patients, perhaps causing part of the relationship between ASA scores and in-hospital complications and resulting in a somewhat overestimated OR. Still, UTI usually do not affect ASA scores and we also found associations between ASA scores and superficial wound infections during the hospital stay which are generally a result of skin incisions during hip fracture surgery.

It must be noted that we employ both logistic regression and survival analyses and that part of the analyses are restricted to a subset of patients. Estimated effect sizes are thus not directly comparable between outcomes. However, the fact that we observe consistent associations between ASA score and various outcomes measured in two different registers and in different study populations lends credibility to our findings. The Swedish NPR has high sensitivity and positive predictive values (PPV) with respect to data on first hip fractures as well as some complications under study, such as myocardial infarction and stroke $[12,13]$ but data quality is somewhat poorer for other outcomes. Still, previous validation studies estimated reasonable PPVs of approximately $80 \%$ for heart failure, venous thromboembolisms, or second hip fractures and it is likely that inpatient registers capture the vast majority of such events [12, 13]. Whether and how the remaining outcome misclassification of these complications is associated with ASA scores and may have affected our estimates is unknown. The sensitivity of pneumonia diagnoses in the NPR is likely much lower [12] but the association between ASA scores and pneumonia was strikingly similar when measured in the NPR and SRH. However, it should also be noted that infection diagnoses in the SRH have not been validated in previous research.

We could not identify complications treated in primary care, but the outcomes included in this study usually require hospitalization. Moreover, ASA scores were not available for $8 \%$ of patients. Additional analyses indicated that the majority of missing ASA scores were clustered at the beginning of the study period and ASA scores have since become mandatory to report. Missing ASA scores were only weakly correlated with 1-year mortality and considerably more common among patients who did not undergo surgery, but this group encompasses only $0.4 \%$ of patients. Finally, our study was based on hip fracture patients identified in the SRH. Although the SRH includes approximately $80 \%$ of hip fracture patients in Sweden, we have shown in a previous study that these patients have somewhat lower short-term mortality rates than the total population of hip fracture patients in Sweden [13]. It is thus likely that the SRH does not include some of the frailest patients who die shortly after their hip fracture. Assuming that these patients would be assigned high ASA scores, it is possible that our findings - to some extent-underestimate the association between ASA score and mortality or post-operative complications.

Our results suggest that patients with high ASA scores deserve further clinical and scientific attention. We found that almost half of patients with ASA score 4 die within 1 year of sustaining their first hip fracture and that notable proportions experience pneumonia, heart failure, and second hip fractures. Several of the complications examined in this study may be preventable through adequate drug treatment, rehabilitation, and risk awareness among patients and clinicians. Future studies should examine the mechanisms linking ASA scores to the risk of different complications in order to improve preventive strategies. In addition, further research should investigate regional differences in the relationship between ASA scores and 
complications after hip fracture since the risk of postoperative complications after hip fracture likely depends on clinical guidelines and management and may hence differ between clinics, regions, and countries.

Supplementary Information The online version contains supplementary material available at https://doi.org/10.1007/s00198-021-05956-w.

Funding Open access funding provided by Karolinska Institute. The results reported herein correspond to specific aims of grant M18-005 to investigator Karin Modig from the Åke Wiberg Foundation. This work was also supported by grants provided by Region Stockholm (ALF project) and grant 2019135 from the Kamprad Family Foundation for Entrepreneurship, Research and Charity.

Data availability Data were provided by the Swedish National Board of Health and Welfare and Statistics Sweden. Restrictions apply to the availability of these data, which are thus not publicly accessible. Data are, however, available from the authors upon reasonable request and with permission of the regional ethics board in Stockholm.

Code availability Statistical code is available upon request from the corresponding author at anna.meyer@ki.se.

\section{Declarations}

Ethics approval This study was approved by the regional ethics committee in Stockholm (permit numbers 2011/136-31/5, 2017/1088-31, and 2018/84-32). The board waived the need for patient consent.

\section{Consent to participate Not applicable.}

Conflicts of interest The authors declare no competing interests.

Open Access This article is licensed under a Creative Commons Attribution-NonCommercial 4.0 International License, which permits any non-commercial use, sharing, adaptation, distribution and reproduction in any medium or format, as long as you give appropriate credit to the original author(s) and the source, provide a link to the Creative Commons licence, and indicate if changes were made. The images or other third party material in this article are included in the article's Creative Commons licence, unless indicated otherwise in a credit line to the material. If material is not included in the article's Creative Commons licence and your intended use is not permitted by statutory regulation or exceeds the permitted use, you will need to obtain permission directly from the copyright holder. To view a copy of this licence, visit http:// creativecommons.org/licenses/by-nc/4.0/.

\section{References}

1. Karampampa K, Ahlbom A, Michaëlsson K, Andersson T, Drefahl S, Modig K (2015) Declining incidence trends for hip fractures have not been accompanied by improvements in lifetime risk or post-fracture survival - a nationwide study of the Swedish population 60 years and older. Bone 78:55-61. https://doi.org/10.1016/j. bone.2015.04.032

2. Meyer AC, Ek S, Drefahl S, Ahlbom A, Hedström M, Modig K (2021) Trends in hip fracture incidence, recurrence, and survival by education and comorbidity: a Swedish Register-based Study.
Epidemiology. 32:425-433. https://doi.org/10.1097/ede. 0000000000001321

3. Doyle DJ, Goyal A, Bansal P, Garmon EH (2021) American Society of Anesthesiologists Classification. In: StatPearls. StatPearls Publishing LLC

4. Söderqvist A, Ekström W, Ponzer S, Pettersson H, Cederholm T, Dalén N, Hedström M, Tidermark J (2009) Prediction of mortality in elderly patients with hip fractures: a two-year prospective study of 1,944 patients. Gerontology 55(5):496-504. https://doi.org/10. $1159 / 000230587$

5. Ekström W, Samuelsson B, Ponzer S, Cederholm T, Thorngren KG, Hedström M (2015) Sex effects on short-term complications after hip fracture: a prospective cohort study. Clin Interv Aging 10:1259

6. Hedström M, Gröndal L, Ahl T (1999) Urinary tract infection in patients with hip fractures. Injury 30(5):341-343. https://doi.org/ 10.1016/S0020-1383(99)00094-7

7. Lv H, Yin P, Long A, Gao Y, Zhao Z, Li J, Zhang L, Zhang L, Tang P (2016) Clinical characteristics and risk factors of postoperative pneumonia after hip fracture surgery: a prospective cohort study. Osteoporos Int 27(10):3001-3009. https://doi.org/10.1007/s00198016-3624-5

8. Ali AM, Gibbons CER (2017) Predictors of 30-day hospital readmission after hip fracture: a systematic review. Injury 48(2):243252. https://doi.org/10.1016/j.injury.2017.01.005

9. Chiang C-H, Liu C-J, Chen P-J, Huang C-C, Hsu C-Y, Chen Z-Y, Chan W-L, Huang P-H, Chen T-J, Chung C-M, Lin S-J, Chen J-W, Leu H-B (2013) Hip fracture and risk of acute myocardial infarction: a nationwide study. J Bone Miner Res 28(2):404-411. https:// doi.org/10.1002/jbmr.1714

10. Pedersen AB, Ehrenstein V, Szépligeti SK, Sørensen HT (2017) Hip fracture, comorbidity, and the risk of myocardial infarction and stroke: a Danish Nationwide Cohort Study, 1995-2015. J Bone Miner Res 32(12):2339-2346. https://doi.org/10.1002/jbmr. 3242

11. Tsai CH, Lin CL, Hsu HC, Chung WS (2015) Increased risk of stroke among hip fracture patients: a nationwide cohort study. Osteoporos Int 26(2):645-652. https://doi.org/10.1007/s00198014-2919-7

12. Ludvigsson JF, Andersson E, Ekbom A, Feychting M, Kim J-L, Reuterwall C, Heurgren M, Olausson P (2011) External review and validation of the Swedish national inpatient register. BMC Public Health 11(1):450. https://doi.org/10.1186/1471-2458-11-450

13. Meyer AC, Hedström M, Modig K (2020) The Swedish Hip Fracture Register and National Patient Register were valuable for research on hip fractures: comparison of two registers. J Clin Epidemiol 125:91-99. https://doi.org/10.1016/j.jclinepi.2020.06. 003

14. Doherty WJ, Stubbs TA, Chaplin A, Reed MR, Sayer AA, Witham MD, Sorial AK (2020) Prediction of postoperative outcomes following hip fracture surgery: independent validation and recalibration of the Nottingham Hip Fracture Score. J Am Med Dir Assoc 22:663-669.e2. https://doi.org/10.1016/j.jamda.2020.07.013

15. Lizaur-Utrilla A, Serna-Berna R, Lopez-Prats FA, Gil-Guillen V (2015) Early rehospitalization after hip fracture in elderly patients: risk factors and prognosis. Arch Orthop Trauma Surg 135(12): 1663-1667. https://doi.org/10.1007/s00402-015-2328-1

16. French DD, Bass E, Bradham DD, Campbell RR, Rubenstein ZL (2008) Rehospitalization after hip fracture: predictors and prognosis from a National Veterans Study. J Am Geriatr Soc 56(4):705-710. https://doi.org/10.1111/j.1532-5415.2007.01479.x

17. Kastanis G, Topalidou A, Alpantaki K, Rosiadis M, Balalis K (2016) Is the ASA score in geriatric hip fractures a predictive factor for complications and readmission? Scientifica 2016:70962457096246. https://doi.org/10.1155/2016/7096245 
18. Ondeck NT, Bovonratwet P, Ibe IK, Bohl DD, McLynn RP, Cui JJ, Baumgaertner MR, Grauer JN (2018) Discriminative ability for adverse outcomes after surgical management of hip fractures: a comparison of the Charlson Comorbidity Index, Elixhauser Comorbidity Measure, and Modified Frailty Index. J Orthop Trauma 32(5):231-237

19. Ranta S, Hynynen M, Tammisto T (1997) A survey of the ASA physical status classification: significant variation in allocation among Finnish anaesthesiologists. Acta Anaesthesiol Scand 41(5): 629-632. https://doi.org/10.1111/j.1399-6576.1997.tb04755.x

20. Ihejirika RC, Thakore RV, Sathiyakumar V, Ehrenfeld JM, Obremskey WT, Sethi MK (2015) An assessment of the interrater reliability of the ASA physical status score in the orthopaedic trauma population. Injury 46(4):542-546. https://doi.org/10.1016/j. injury.2014.02.039
21. Ji H-M, Han J, Bae H-W, Won Y-Y (2017) Combination of measures of handgrip strength and red cell distribution width can predict in-hospital complications better than the ASA grade after hip fracture surgery in the elderly. BMC Musculoskelet Disord 18(1):375375. https://doi.org/10.1186/s12891-017-1738-3

22. Hedström M, Ljungqvist O, Cederholm T (2006) Metabolism and catabolism in hip fracture patients: nutritional and anabolic intervention - a review. Acta Orthop 77(5):741-747. https://doi. org/10.1080/17453670610012926

Publisher's note Springer Nature remains neutral with regard to jurisdictional claims in published maps and institutional affiliations. 